2015-03-01

\title{
Subsea cable tracking by an unmanned surface vehicle
}

\section{Szyrowski, T}

http://hdl.handle.net/10026.1/3644

\subsection{3/ut.32.217}

Underwater Technology: International Journal of the Society for Underwater Society for Underwater Technology

All content in PEARL is protected by copyright law. Author manuscripts are made available in accordance with publisher policies. Please cite only the published version using the details provided on the item record or document. In the absence of an open licence (e.g. Creative Commons), permissions for further reuse of content should be sought from the publisher or author. 


\title{
Subsea cable tracking by an unmanned surface vehicle
}

\author{
Tomasz Szyrowski*1, Amit Motwani ${ }^{1}$, Sanjay K Sharma ${ }^{1}$, Robert Sutton ${ }^{1}$ and Gareth A Kennedy ${ }^{2}$ \\ ${ }^{1}$ School of Marine Science and Engineering, Plymouth University, Plymouth, UK \\ ${ }^{2}$ Camborne School of Mines, Exeter University, Cornwall Campus, Penryn, UK
}

Received 29 July 2014; Accepted 4 December 2014

\begin{abstract}
Subsea cable localisation is a demanding task that requires a lot of time, effort and expense. In the present paper the authors propose a methodology that is automated and inexpensive, based on magnetic detection from a small unmanned surface vehicle (USV) and the use of a batch particle filter (BPF) algorithm. A dynamic path planning algorithm for the USV is also developed so that adequate samples of the magnetic field readings can be gathered for processing by the BPF. All of these elements work together online as the cable is tracked, which was demonstrated in a simulated mission.
\end{abstract}

Keywords: batch particle filtering, subsea cable tracking, weighted interval kalman filtering, fuzzy logic

\section{Introduction}

Technological advancement has brought with it an increasing number of maritime transportation links and offshore structures, and these are set to increase. For example, the UK government has recently announced plans to develop what will be the world's largest offshore wind farm off the coast of Suffolk, UK (Guardian, 2014). Such farms require arrays of subsea cables to link each of the turbines together as well as to offshore substations. Detection of such cables is important for periodical inspection and maintenance.

Cable detection methods that are presently used have come a long way, but their scope is still fairly limited. Optical inspection, hydroacoustic localisation and magnetic detection are the three main methods for subsea surveys. The main limitation of

* Contact author. Email address: tomasz.szyrowski@plymouth.ac.uk the first two are that they fail to detect cables that are buried or hidden under plant growth, as is often the case in coastal and shallow waters (Szyrowski et al., 2013a).

Detection of subsea ferromagnetic objects is mainly based on mathematical inversion methods (Cowls and Jordan, 2002; Won, 2003). Two or more magnetic detectors separated some distance from one another are used to measure the magnetic field (MF) emitted by the ferromagnetic object or induced current, whereby its location can be determined through triangulation. However, this method is only accurate up to a range of 3m (Takagi et al., 1996; Kojima et al., 1997; Szyrowski et al., 2013b). This reduced range translates into requiring a diver or expensive underwater vehicle to perform the survey.

In the present paper, a solution to this problem is proposed whereby the detection of a subsea cable is carried out by an autonomous vehicle from the surface. To achieve this, the authors have developed a precise vehicle navigation system, as well as a guidance algorithm that directs the vehicle along required pathways that enable a stream of measurements of the MF to be collected. From this data, a novel localisation algorithm based on particle filtering $(\mathrm{PF})$ is applied to determine the location of the source of the MF.

Instead of using a single measurement from each of the distance-separated magnetic detectors and then trying to determine the source by inversion methods, the batch particle filter (BPF) uses several measurements of the MF taken in the vicinity of the source. In order to acquire these, the unmanned surface vehicle (USV) inspects the area where the cable is thought to lie. Once a meaningful reading of an MF is obtained, a specific survey 
path that crosses the cable is dynamically planned and further measurements are gathered.

A fuzzy logic algorithm is used to distinguish between meaningful readings that originate from the MF induced by the cable and the surrounding magnetic noise. When the cable has been crossed and no more meaningful readings are obtained, the set of readings is given to the BPF, which then estimates the exact crossover point of the cable based on all the readings obtained. Using this information, the position of the cable some distance downstream is estimated, and the vehicle's survey path is re-planned.

The USV must be able to trace the planned survey path and, above all, localise itself and accurately determine its heading at every instant. The precise localisation not only enables smooth autonomous navigation, but also gives the MF readings taken along directions relative to the vehicle's own heading. In order to compare the successive readings, they must all be described in a common global reference frame before using them to determine the magnetic source. Hence, a novel, robust heading estimation technique based on interval Kalman filtering has been applied to estimate the USV's heading.

The BPF algorithm has been tested offline on real subsea cable survey data gathered during a manned expedition. Although the MF in this case was induced by an alternating current flowing through the cable, the method can be applied to localising any ferromagnetic object by equipping the USV with an eddy current inducing coil for generating a magnetic response in the object. The integration of the BPF source estimation method with autonomous USV navigation and path planning has been tested on computer simulations, with sea trials being planned for the future.

This approach offers advantages with respect to conventional methods, for example, enabling operations in hazardous environments without risking divers' safety or needing to employ manned ships. Moreover, autonomous tracking of the dynamically planned path is carried out accurately and efficiently, avoiding delays in the control loop that would inevitably exist in relaying information continuously regarding the path updates to the crew of a manned vessel, saving both time and costs.

The rest of the present paper is organised as follows: the BPF algorithm and its motivation as an efficient tool for subsea ferromagnetic object localisation is presented in section 2. The fuzzy logic algorithm used for discriminating meaningful readings from noise is also described in section 2. Section 3 then discusses the autonomous operation of the USV, with emphasis on the robust heading estimation procedure using the so called weighted interval Kalman filter (WIKF). Section 4 details the simulation of a cable-tracking mission, and finally conclusions and future objectives are discussed in section 5 .

\section{Selective batch particle filtering for ferromagnetic object localisation}

In order to detect ferromagnetic objects, these must emit an MF. An MF in ferrous objects originates from electric currents flowing within them. In objects such as cables or pipes, an electric current can be injected, thereby producing an MF. Where this is not possible, an alternating MF generated by an external agent can induce eddy currents in the body of the object, which then emits a secondary MF that can be detected (Cowls and Jordan, 2002; Tumanski, 2007).

In the maritime subsea environment, the propagation of the MF is highly affected by the conductivity of the sea water, which depends on its salinity (Bogie, 1972; King, 1989). Currently used methods for subsea cable localisation, which are short range and applicable only within distances of up to $3 \mathrm{~m}$ from the source, assume that the conductivity of the water is uniform and can be neglected (Szyrowski et al., 2013b). They also assume the MF decay, as a function of the distance from the source, follows a simple decay function, and hence the difference between two readings separated by a known distance allows inference of the distance of the readings from the source. However, Cowls and Jordan (2002) have pointed out that this assumption is not always true: although the signal strength generally decays as $r^{-3}$, it can include some variation and can be difficult to calculate precisely.

The strength of the MF generated by a subsea cable on the water surface can be modelled as a scalar field, as shown in Fig 1. In the case of a cable with small curvature, the MF generated by the cable at any point in space $\left(P_{k}\right)$ can be approximated by that owing solely to the point of the cable $\left(S_{k}\right)$ that is closest to $P_{k}$, i.e.:

$$
B_{\mathrm{k}}=\frac{\mu_{0} I}{a 4 \pi r_{k}^{3}}\left(d_{k} \times\left[P_{k}-S_{k}\right]\right)
$$

where $d_{k}$ is the direction of the cable at point $S_{k} ; r_{k}$ is the shortest distance to the cable; $a$ is an attenuation parameter; $\mu_{0}$ is the permeability of free space; and $I$ is the electric current. In a small area, the parameter $(a)$ can be considered to represent an averaged attenuation; hence, for a short section of cable, the term $\frac{\mu_{0} I}{a 4 \pi}$ can be grouped into a single constant $(c)$. Note that the contribution to the MF at $P_{k}$ is mostly owing to the section of cable around 


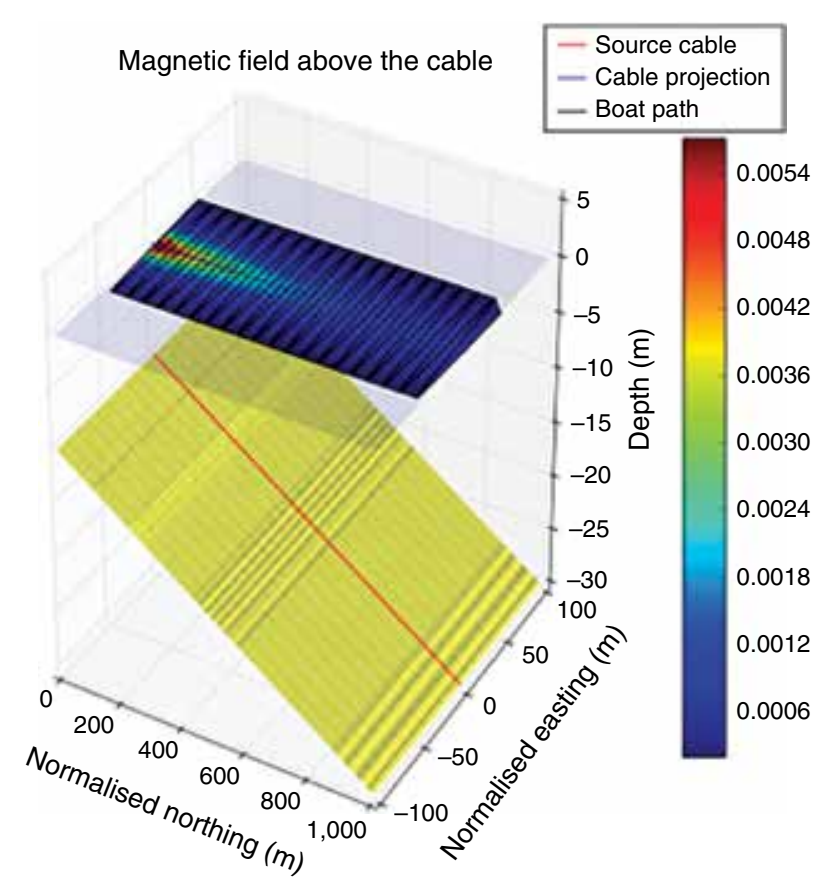

Fig 1: Distribution of magnetic field from long ferromagnetic wire

$S_{k}$, and is not much affected by portions of the cable further away where the attenuation might be different. Hence, MF can be described as:

$$
B_{\mathrm{k}}=c \frac{\left(d_{k} \times\left[P_{k}-S_{k}\right]\right)}{r_{k}^{3}}
$$

The problem of determining the location of the source $\left(S_{k}\right)$ from measurements of the MF at various sample points also must include estimating the local value of the parameter $c$. This problem can be thought of as a regression of the various measurements of the MF vector at various sample points on the sea surface onto the model described

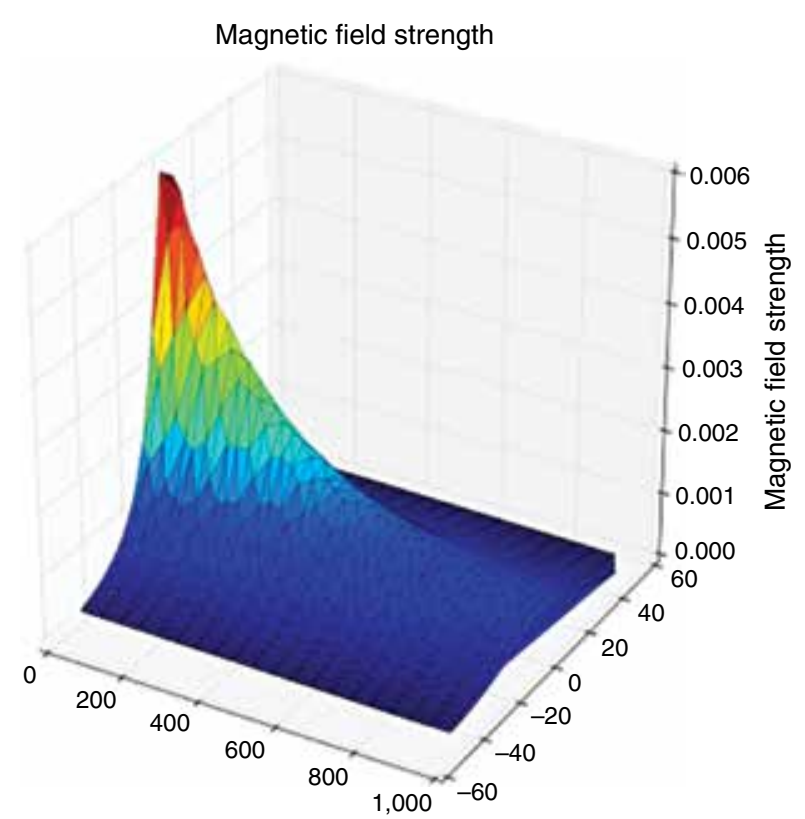

by Equation 2 parameterised by source points $\left(S_{k}\right)$, direction vectors $\left(d_{k}\right)$ and the averaged attenuation (c) (Fig 2).

If it can be considered that the sample points all correspond to the same source (i.e. that they are obtained from crossing the cable perpendicularly), then the problem is simplified to determining one source (in addition, to the average attenuation $(c)$ ). This scenario is depicted in Fig 3, in which the plane $\left(\Pi_{k}\right)$ is perpendicular to the cable at the intersection point $\left(S_{k}\right)$. Then for each sample point $\left(P_{k}\right)$ on the surface along the line $\left(l_{k}=W \cap \Pi_{k}\right), S_{k}$ is the closest point of the cable to it. In this case, the MF vector $B_{k}$ at each $P_{k}$ is contained within the plane $\Pi_{k}$.

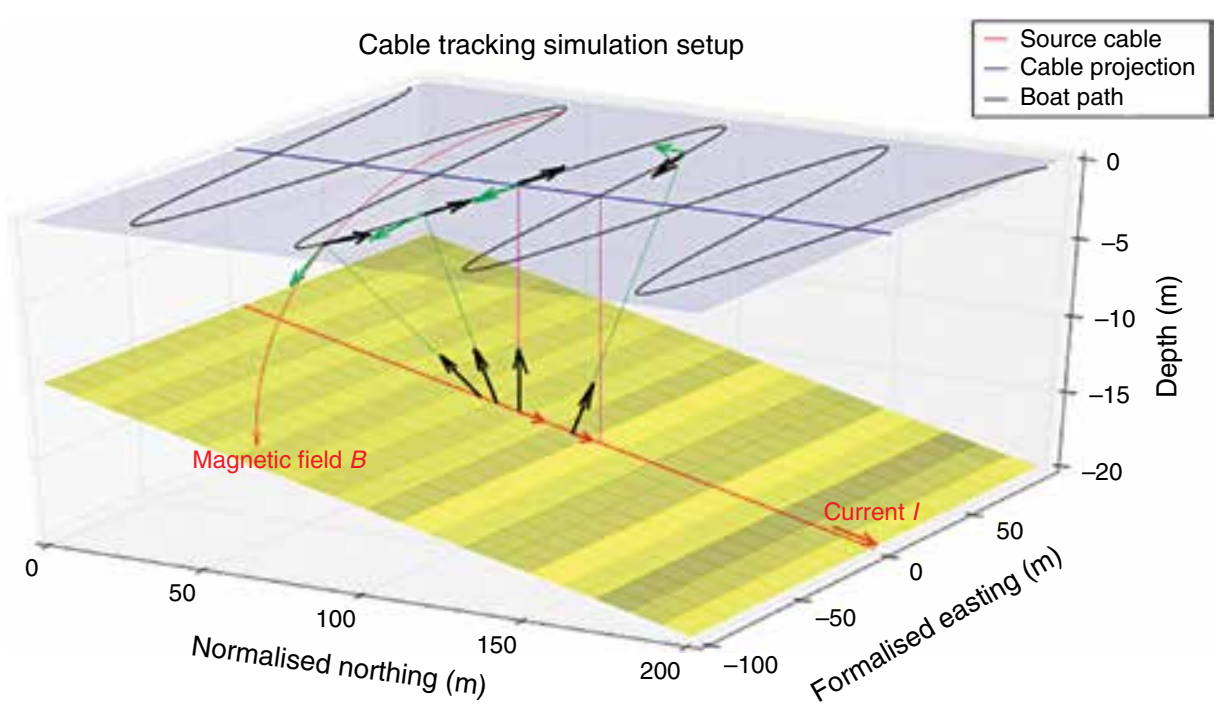

Fig 2: MF vector field in sample points 


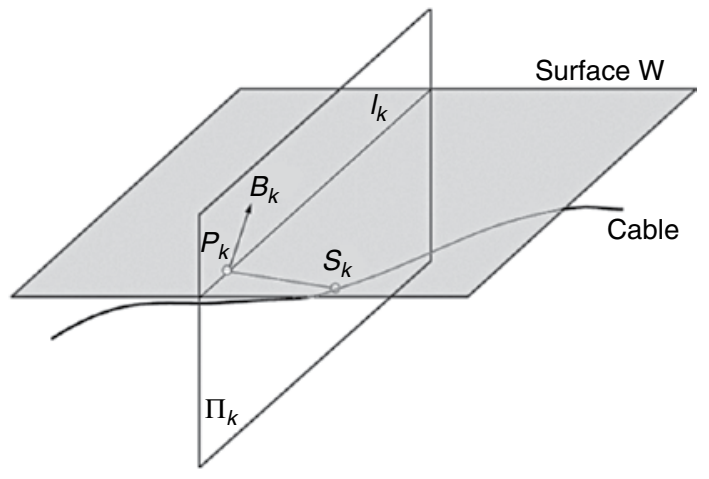

Fig 3: Basic cable-tracking parameters

The successive MF measurements along $l_{k}$ from a horizontal coil whose axis is aligned with the direction of $l_{k}$ are then established. The readings are generated according to Equation 2 for simulation purposes, using values of current and attenuation measured from previously conducted trials on the Baltic Sea (Szyrowski et al., 2014), and generated at regular intervals along $l_{k}$. This is consistent with taking samples at regular time intervals from the USV moving along $l_{k}$ at constant speed.

The coil readings can be expressed as:

$$
z_{k}=h\left(x_{k}\right)+v_{k}=\left[\begin{array}{l}
C_{H}+v_{k}^{H} \\
C_{V}+v_{k}^{V}
\end{array}\right]=\left[\begin{array}{l}
B_{k} \cdot h_{k}+v_{k}^{H} \\
B_{k} \cdot z+v_{k}^{V}
\end{array}\right]
$$

where $\left(C_{H}+v_{k}^{H}\right)$ corresponds to the output from the horizontal coil, and $\left(C_{V}+v_{k}{ }^{V}\right)$ corresponds to the output from the vertical coil; $v_{k}^{H}$ and $v_{k}{ }^{V}$ are random measurement noises associated with the readings; $h_{k}$ represents a unit-length vector in the direction of the horizontal coil axis, which is assumed to be the same as the direction of the line $\left(l_{k}\right)$; and $z$ is a vertical unit-length vector. The total MF is the vector sum of these two components.

The coil measurements in terms of the locations of the sampling point, cable source and the average attenuation parameter can be obtained simply by substituting Equation 2 into Equation 3, and is given by:

$$
z_{k}=\left[\begin{array}{c}
c \frac{\left(d_{k+1} \times\left[P_{k}-S_{k}\right]\right)}{\left.I\left[P_{k}-S_{k}\right]\right]^{3}} \cdot h_{k}+v_{k}^{H} \\
c \frac{\left(d_{k+1} \times\left[P_{k}-S_{k}\right]\right)}{\left.I\left[P_{k}-S_{k}\right]\right]^{3}} \cdot\left[\begin{array}{lll}
0 & 0 & 1
\end{array}\right]+v_{k}^{V}
\end{array}\right]
$$

Having assumed that $d_{k}$ is perpendicular to $\Pi_{k}$ - i.e. that the vehicle moves along a path perpendicular to the direction of the cable - Equation 4 reduces to:

$$
z_{k}=\left[\begin{array}{l}
\left|B_{k}\right| \cos (\theta)+v_{k}^{H} \\
\left|B_{k}\right| \sin (\theta)+v_{k}^{V}
\end{array}\right]
$$

where $\theta$ is the angle between $B_{k}$ and $l_{k}$. Equation 5 is used in the implementation of a BPF to estimate the source $S_{k}$ for a single crossover line $l_{k}$.

Particle filters (PF), introduced by Gordon (1993), are a tool to estimate the posterior probability density function (PDF) of state variables from observations (Crisan and Obanubi, 2012; Fallon and Godsill, 2010). PFs use a recursive scheme to approximate the PDF by a set of random samples called particles, which tend to concentrate in regions of high probability density, serving to approximate the true PDF. The operation of the PF algorithm is schematically represented in Fig 4 . An initialisation step generates random particles representing a hypothetical cable location and an attenuation parameter. The particles have a weight assigned to them on the basis of the distance, or error, between the hypothetical MF they represent and the experimental data. The particles are resampled and regenerated until they converge to the area which guarantees a satisfactory error. (The reader interested in a more detailed description of PF may consult Szyrowski et al. (2014).)

In the BPF algorithm, each particle, which is representative of a possible location of the cable source, has an associated weight or probability, and the most plausible location is given by the particle with the largest weight. The rest of the particles in the set tend to cumulate around this solution. In a regeneration stage, only a quarter of the particles, those with the highest weights, are maintained. Each one

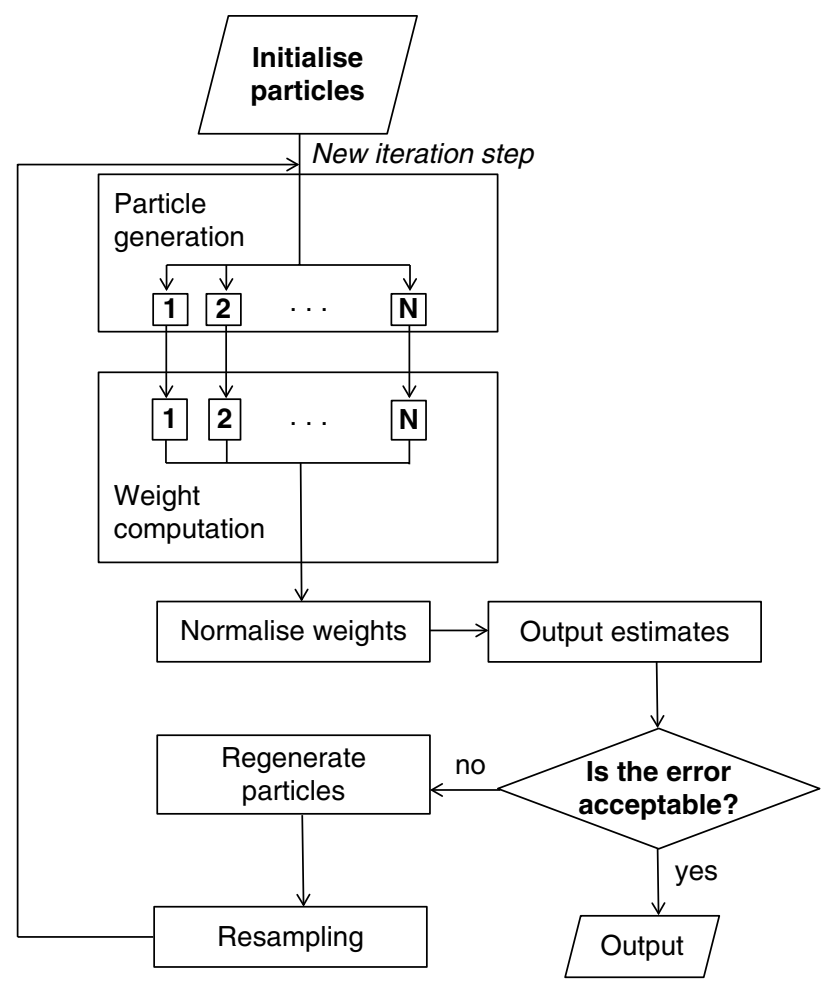

Fig 4: Particle filter algorithm 
Fuzzy logic distrimination of data points and seting ROI for particle filtering

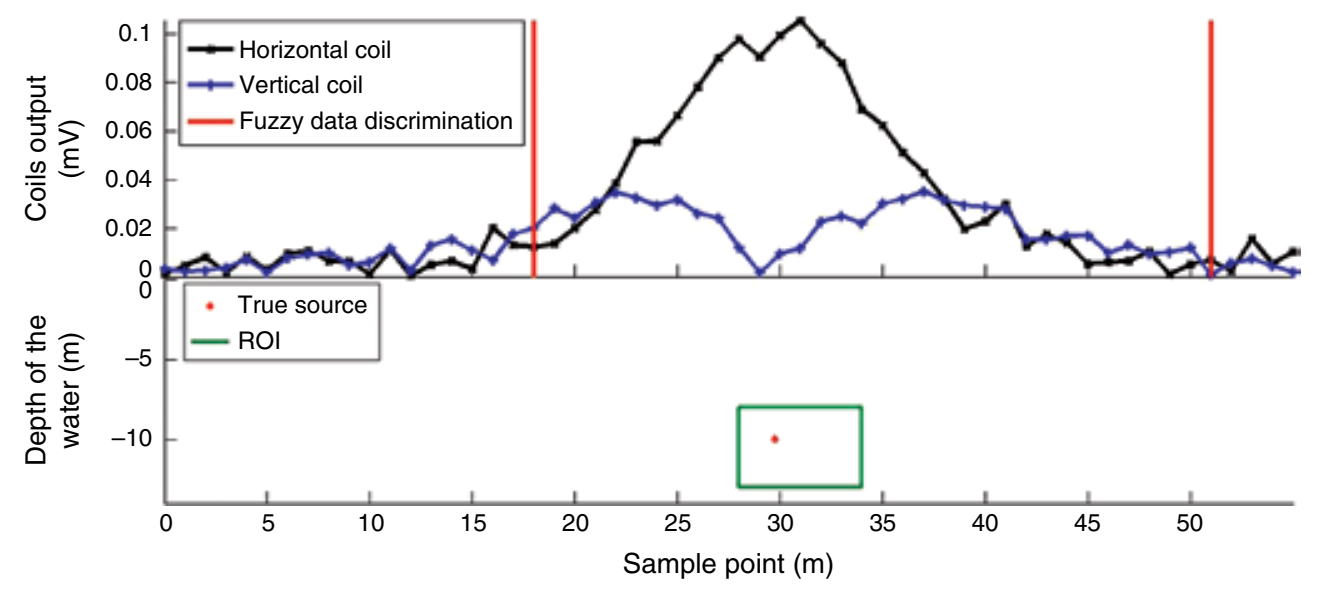

Fig 5: Region of interest $\mathrm{ROI}$ for MF source detection

of these particles then generates three new particles randomly in the space surrounding it. The higher the weight of the mother particle, the smaller is the surrounding area in which the new particles are allowed to be generated. Thus, particles with smaller weights will have their children cover a wider area, whilst those particles with high weights will conceive particles that are very close to themselves. After the regeneration step, the algorithm starts a new iteration where for each particle, the MF and the resulting theoretical coil readings are generated. According to how well each of these fit the actual readings, their weights are then computed.

In order to start the algorithm, a set of initial particles must be chosen. First, a region of interest (ROI) is established. Then, the horizontal coil readings of the MF should, in theory, reach a peak value when the reading is taken directly above the cable - that is, when the path $\left(l_{k}\right)$ apparently intersects the cable when viewed from above. At this point, the theoretical reading from the vertical coil should be zero. Based on this observation, the ROI is chosen as a rectangular area below the sample point with the maximum difference between horizontal and vertical coil reading. In practice, this reading might not necessarily correspond to a measurement taken directly above the cable, as the readings incorporate a stochastic measurement noise. Thus, the ROI is extended so as to cover a horizontal distance corresponding to the abscissas of the five previous and five posterior measurement locations as well.

During an actual survey, the depth of the water column is measured with an echo-sounder at each sample point. It is conceivable that the cable could be buried up to $3 \mathrm{~m}$ under the seabed, and that certain sections may be suspended in the water above it. Hence, the height of the ROI is taken from $3 \mathrm{~m}$ below the seabed to $2 \mathrm{~m}$ above it. In the simulation carried out herein, the seabed was assumed to lie at a constant $10 \mathrm{~m}$ below the surface. For one crossover line $\left(l_{k}\right)$, the simulated coil readings of the MF and the ROI established from these are depicted in Fig 5 .

Once the ROI has been established, $N$ particles are generated randomly within it. In the simulation carried out, the number of particles was set to $N=100$. The locations of the initial particles are shown in Fig 6 . Each of these represents a hypothetical location of the cable source. In addition, to each of these particles an averaged attenuation parameter $c$ is also assigned. For the BPF algorithm, each particle is assigned a value of $c$ randomly chosen within a range of $10 \%$ of the nominal value set experimentally.

Each of the particles is then assigned a weight. In order to do this, the theoretical coil readings for each particle (hypothetical cable source location and parameter $c$ ) are generated according to Equations 2 and 5 . The mean square error between these and the actual readings is computed, normalised and assigned to the corresponding particle. This completes the initialisation of the BPF algorithm.

The BPF then takes on an iterative character. In each iteration, $75 \%$ of the particles are regenerated from the $25 \%$ with the largest weights. For each of these progenitor particles, three new particles are generated by adding Gaussian noise to their position and value of $c$. The amplitude of the noise added is inversely proportional to the engendering particle's weight. The particle with the largest weight (and hence whose MF distribution along the sampling points coincides most closely to the measured one) generates particles closer to itself than do those particles with a smaller weight that are not quite so close to the true source, and hence spread their seeds further afar. 


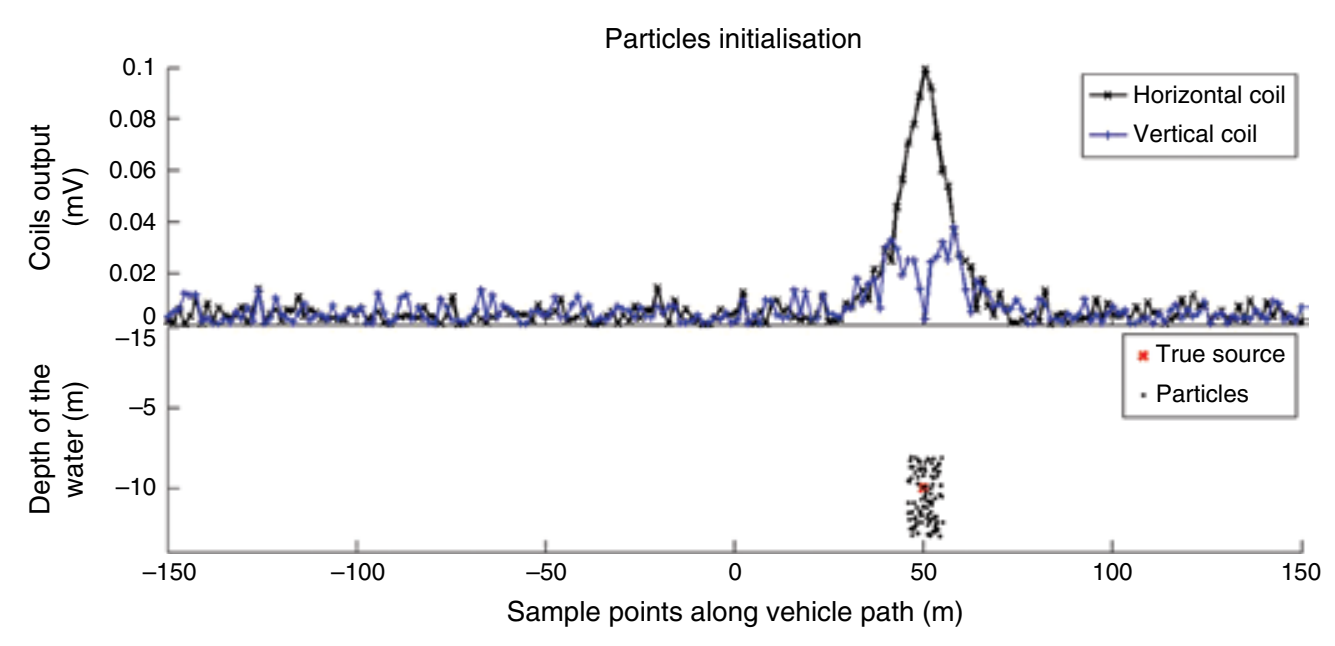

Fig 6: Initialisation of particle representing the hypothetical source

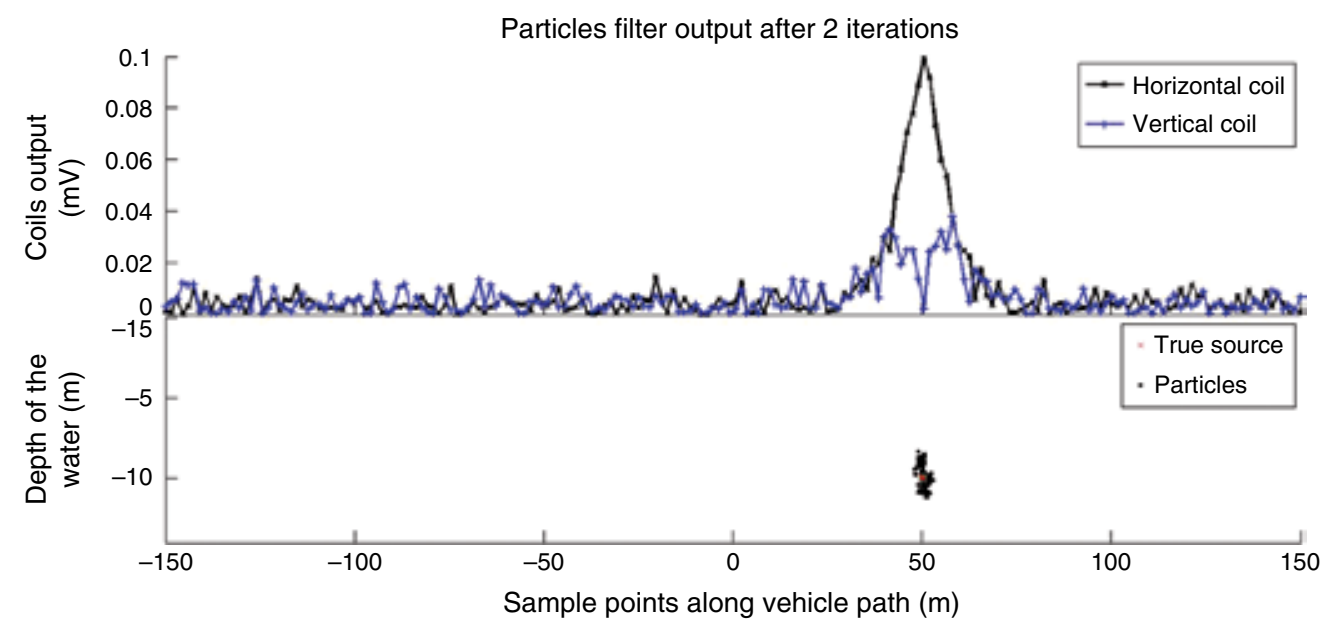

Fig 7: Particles after resampling

The new set of particles then undergoes the same procedure of weight assignment, and the whole process is repeated. During this process, the particles tend to cumulate in the region of highest probability of the location of the true source. It should be recalled that each of the particles also represents a certain value of the parameter $(c)$. The particle with the highest weight represents not just the location of the true source, but also the actual value of the parameter $(c)$. Hence, the BPF algorithm gives not only an estimate of the most likely location of the true cable source, but also of the locally averaged attenuation rate. One of the key advantages of this procedure is that it is able to locate the source even without a correct prior estimate of the parameter $(c)$, which is not possible using other well-known techniques such as those based on Kalman filtering.

Observation of Figs 5 to 8 reveals that much of the coil readings actually correspond to background magnetic noise rather than to any meaningful measurements of the MF emitted by the cable. In order to both reduce the computational burden on the
BPF and increase its efficiency, it becomes necessary to filter out the unwanted readings. This is accomplished with discriminatory filter based on fuzzy logic decision-making. It is based on observing that the measurements are meaningful when the magnitudes of the readings are consistently larger than some threshold value. Based on this, in order to filter out some of the noise from the readings, a simple moving average (SMA) of both the horizontal and vertical coil readings is computed. The average of these two SMAs is then obtained and used as input to the membership functions depicted in Fig 9.

The fuzzy decision-making is based on the following rules:

- Rule 1: If SMA negative, then $f f$ is negative;

- Rule 2: If SMA is zero, then $f f$ is zero; and

- Rule 3: If SMA is positive, then $f f$ is positive;

where the variable $f f$ is the output of the fuzzy classifier, and its crisp value is obtained from the output membership functions shown in Fig 10. 


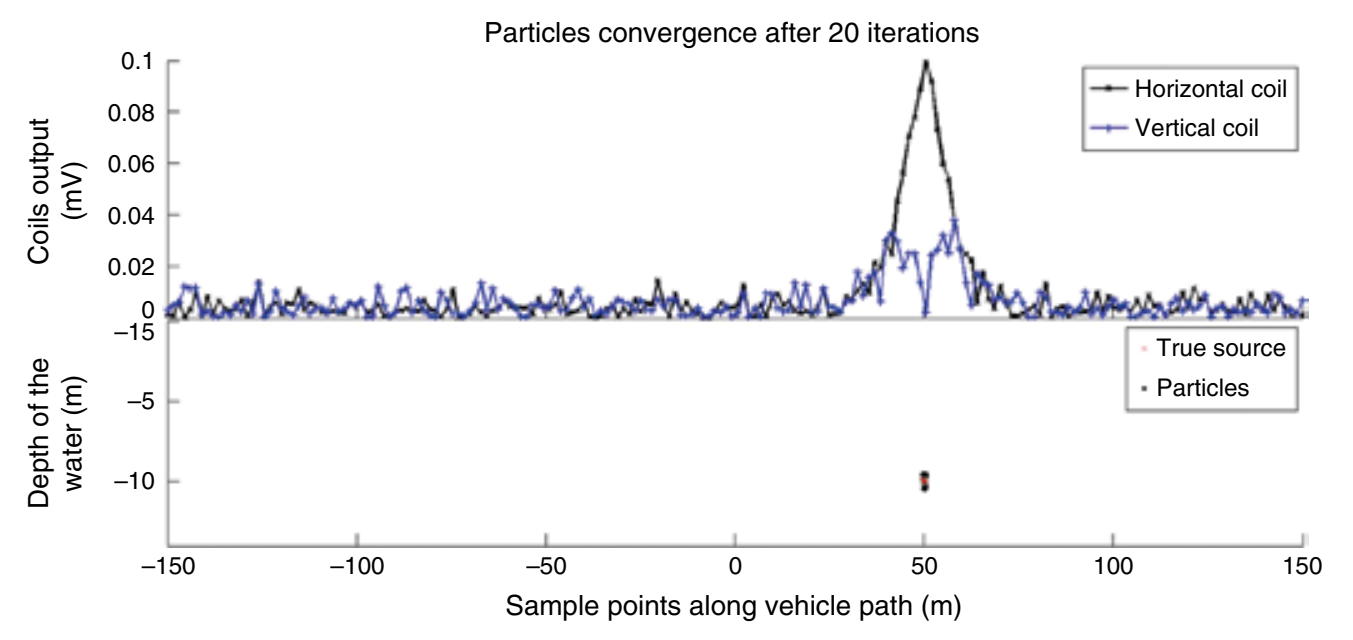

Fig 8: Convergence of particles after 20 iterations

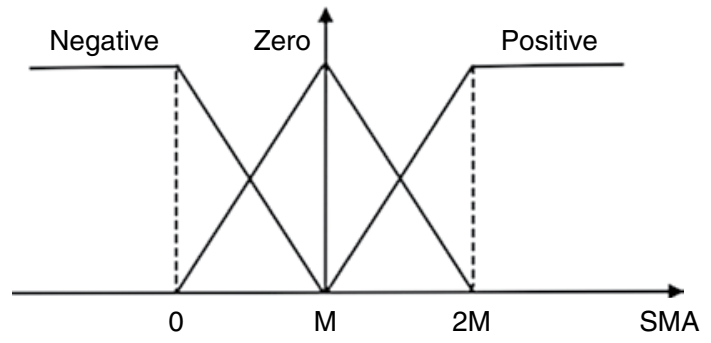

Fig 9: Input membership functions

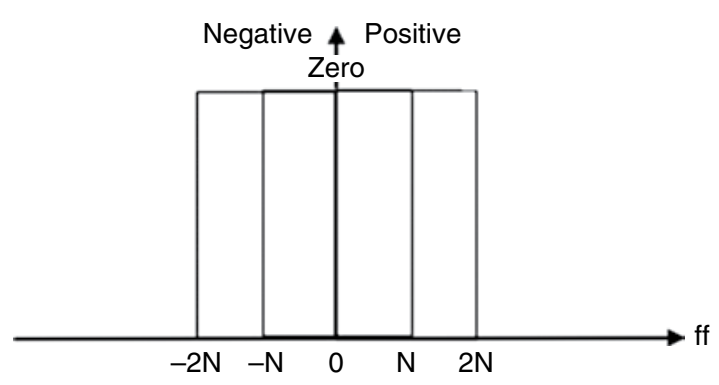

Fig 10: Output membership functions

The value of $f f$, or fuzzy flag, is used as a flag to indicate whether the coil readings are meaningful. Only those readings for which $f f$ is greater than zero are considered by the PF. The two vertical lines in Fig 5 represent the range in which the fuzzy flag is positive for the readings shown in the graph.

The value of the fuzzy flag $f f$ is used to indicate when the coil readings are meaningful. It is initially set to zero, as the first readings are taken at the start of a crossover line $\left(l_{k}\right)$. When the vehicle is within a certain distance of the cable and the readings start to increase, the flag changes to 1 , indicating that these readings should be stored and later passed on to the BPF. Eventually, after the vehicle has crossed the cable, the flag returns to zero, and the stored readings are sent to be processed by the BPF. The instants at which the flag is raised and then lowered again are shown as two vertical red lines in Fig 5. As will be described in the next section, the flag also serves a dual purpose, which is to indicate when the USV may move onto the next cable crossover line.

\section{Autonomous surface vehicle navigation}

The number and type of potential applications for autonomous USVs has increased dramatically in recent times with the availability of low-cost sensing devices (Motwani, 2012). The Springer USV, shown in Fig 11 (Naeem et al., 2006), is a 4m-long twinhull catamaran built at Plymouth University in the UK and is one such vehicle that is being used as a test-bed for developing robust intelligent navigation, guidance and autopilot systems. In particular, a novel heading estimation technique developed for Springer is described and used herein for underwater cable localisation and tracking.

Accurate heading estimation is, of course, important for autonomous navigation, since the vehicle's autopilot acts on it to steer the vehicle onto the desired course. However, it is of particular importance for the cable localisation method described in the

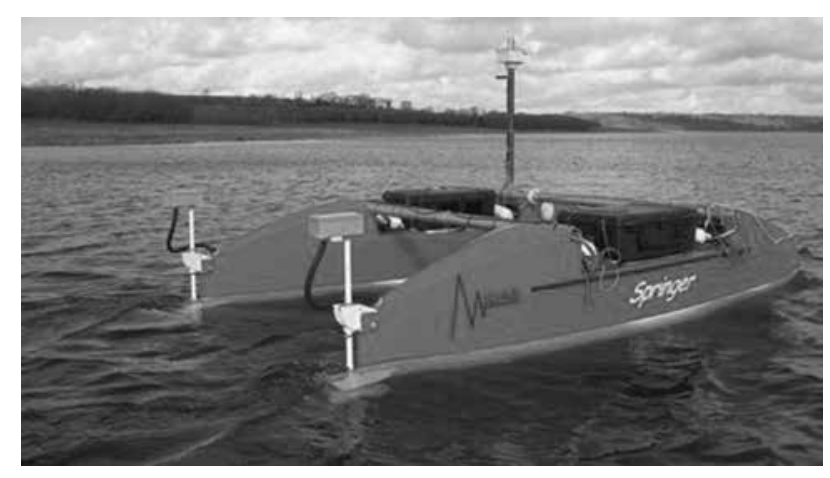

Fig 11: The Springer USV during autonomous navigation trials 
present paper. This is because the measurement of the MF obtained from the horizontal coil depends on the direction of its axis, and so this direction (which could be chosen to coincide with that of the vehicle's heading if the coil is mounted parallel to the vehicle's longitudinal axis) needs to be known.

For localisation, the Springer uses a GPS receiver, while the heading estimation system described here uses a low-cost microelectromechanical system (MEMS) gyroscope and a dynamic steering model of the vehicle. The gyroscope provides a measurement of the vehicle's turning rate, which is subject to some measurement noise and can be used on its own to determine the heading of the vehicle. However, successive integration of the gyroscope output eventually leads to integration drift.

As mentioned, a dynamic steering model of the vehicle was also used. The propulsion of the vehicle is based on two battery-driven trolling motors, one on each hull. Steering of the vehicle is controlled by applying a difference in motor speeds, whereas the overall speed of the vehicle can be changed by varying the average speed of the two motors. Trials have been conducted wherein, maintaining a constant speed, the vehicle was made to carry out various turning manoeuvres. Data of the applied differential motor speed and vehicle turning rate were recorded, and through system identification (SI) models of the form in Equations 6 to 8 were obtained. Basically, the model allows one to predict the turning rate of the vehicle based on the applied differential motor speed over time. In particular, for a constant vehicle speed of $1 \mathrm{~ms}^{-1}$, the model obtained is characterised by the values given in Equation 8:

$$
\begin{aligned}
& x(k+1)=\mathrm{A} x(k)+\mathrm{B} u(k)+\omega(k) \\
& y(k)=\mathrm{C} x(k)+\mathrm{D} u(k) \\
& \mathrm{A}=\left[\begin{array}{cc}
0.8 & -0.2225 \\
1 & 0
\end{array}\right], \mathrm{B}=\left[\begin{array}{l}
1 \\
0
\end{array}\right], \\
& C=\left[\begin{array}{cc}
0 & 0.004225
\end{array}\right], \mathrm{D}=0 ; \mathrm{T}_{s}=1 \mathrm{~s}, \\
& \omega \sim N(0, \mathrm{Q}) ; \mathrm{Q}=\operatorname{cov}(\omega)=0.01 \times \operatorname{diag}\{1,1\}
\end{aligned}
$$

where $u(k)$ is the differential motor speed input in rpm, and $y(k)$ is the rate of change of heading of the vehicle; $\omega$ represents a random input disturbance; and $T_{s}$ is the sampling period. In addition, actuator limitations impose the following constraints on the above model: $|u(k)| \leq 1,200 \mathrm{rpm}$ and $|\Delta u(k)| \equiv|u(k)-u(k-1)| \leq 500 \mathrm{rpm}$.

Both the steering model and gyroscope readings can be used to determine the current heading of the vehicle. However, they can be combined in a Kalman filter (KF) for improved accuracy. Based on the vehicle dynamic model Equations 6 to 8 and the gyro measurement, precise to $0.1 \mathrm{deg} \cdot \mathrm{s}^{-1}$ root mean square (RMS) at $1 \mathrm{~Hz}$ sampling, i.e. with measurement $z(k)$ according to:

$$
\begin{gathered}
z(k)=y(k)+v(k) ; v \sim N(0, R) ; \\
R=\operatorname{cov}(v)=0.1^{2} \operatorname{deg}^{2} s^{-2}
\end{gathered}
$$

The KF estimate of the state vector $\hat{x}(k)$ is obtained by applying Equations 10 to 14 given initial estimates of $\hat{x}$ and error covariance $P(k) \stackrel{\text { def }}{=} \operatorname{var}(x(k)-\hat{x}(k))$ (Simon, 2006):

\section{Prediction:}

$$
\begin{aligned}
& \hat{x}(k \mid k-1)=\mathrm{A} \hat{x}(k-1 \mid k-1)+\mathrm{B} u(k-1) \\
& \mathrm{P}(k \mid k-1)=\mathrm{AP}(k-1 \mid k-1) \mathrm{A}^{\mathrm{T}}+\mathrm{Q}
\end{aligned}
$$

Kalman gain:

$$
\mathrm{K}(k)=\mathrm{P}(k \mid k-1) \mathrm{C}^{\mathrm{T}}\left\{\mathrm{C} \mathrm{P}(k \mid k-1) \mathrm{C}^{\mathrm{T}}+\mathrm{R}\right\}^{-1}
$$

\section{Correction:}

$$
\begin{gathered}
\hat{x}(k \mid k)=\hat{x}(k \mid k-1)+\mathrm{K}(k)[z(k)-\mathrm{C} \hat{x}(k \mid k-1)] \\
\mathrm{P}(k \mid k)=[\mathrm{I}-\mathrm{K}(k) \mathrm{C}] \mathrm{P}(k \mid k-1)
\end{gathered}
$$

The heading of the vehicle can then be obtained from the KF estimate of the state vector as follows:

$$
\hat{\theta}(k)=\hat{\theta}(k-1)+\mathrm{C} \hat{x}(k-1)+\mathrm{D} u(k-1)
$$

The estimated heading is used by the vehicle's autopilot to generate the required differential propeller speed to steer the vehicle along the desired path. The vehicle's guidance system, in turn, generates and updates the reference path of the vehicle via a series of waypoints. A detailed description of the waypoint tracking and autopilot systems used for the autonomous operation of Springer can be found in Annamalai et al. (2014).

Although the use of a KF avoids gyro integration drift and provides a theoretically optimal estimate of the heading (in a statistical sense), it fails if the vehicle model does not accurately reflect the true dynamics of the vehicle (Motwani et al., 2013). In the case presented here, accurate modelling of the steering dynamics via SI is utopian, and at best small modelling errors will arise owing to inhomogeneous sea and wind conditions, variations in payload, etc.

This difficulty is addressed by applying what is known as a weighted interval KF (WIKF), developed for systems with finite modelling uncertainty (Motwani et al., 2014b). Consider a system model, such as Equations 6 to 8 , in which the model coefficients (elements of the matrices A, B, C and D) are not known precisely, 
but are known to lie within certain bounds. Then, describing these coefficients by intervals rather than point-values, the resulting model is called an interval model. Based on this concept, the interval KF (IKF) was proposed as an extension to the $\mathrm{KF}$ for interval systems (Chen et al., 1997). The IKF equations mirror those of the standard KF, but operate on interval values instead using interval arithmetic. The state estimates provided by the IKF are also in the form of intervals rather than point-values.

Although the IKF provides optimal state estimates of interval systems, in practice, a single value is required that most closely matches the state of the true system. The technique used by the WIKF is to obtain this estimate as a weighted average of the IKF bounds. This weight in turn is predicted at each time-step by an adequately trained artificial neural network (ANN) from the sequence of residual data of a standard KF (Motwani et al., 2014a).

Firstly, one or several training missions are devised in which the USV dynamics used to simulate the vehicle's motion is made to vary within certain bounds. The bounds are those of the interval model being proposed. Two KFs are simulated to obtain estimates during these missions. The first is a KF that uses a nominal point-valued model contained in the interval model. The second is a KF that uses the true vehicle dynamics at each instant (i.e. the dynamics used to simulate the vehicle's motion). Because the latter is an ideal KF, its innovations comprise a white noise sequence. However, those of the former digress from being white insofar as the model used differs from the true vehicle dynamics.

In addition, an IKF is also simulated based on the interval model. It can be shown that the ideal KF estimate can be retrieved as a weighted average of the IKF estimate bounds, and this desired weight is calculated and stored at each time-step. Finally, an ANN is trained to match the innovations of the first KF during the mission with the desired weighting sequence. It has been shown that such a trained network can be used to predict adequate weights independently of the true vehicle dynamics and KF nominal model selected for generating the training data, as long as they lie within the interval model that describes the bounded uncertainty. A detailed account of the WIKF can be found in Motwani et al. (2014a).

The navigational effectiveness of the WIKF over the use of a standard KF for uncertain systems will be shown in the next section on the cable-tracking mission.

\section{Cable source tracking}

To track a subsea cable from the USV, the objective is to criss-cross it at right angles. In each crossover line, the MF is measured from the coils, and the BPF described in section 2 is applied to determine the location of the source below that crossover line. The vehicle then advances to the next crossover line, and again the source location below that line is determined. From a navigational point of view, the problem of projecting these crossover lines needs to be addressed.

From an initial estimate of a point of the cable $\left(\hat{S}_{1}\right)$ and an initial estimate of the cable's direction at that point $\left(\hat{d}_{1}\right)$, the first crossover line $\left(l_{1}=\overline{P_{1} Q_{1}}\right)$ is projected as a segment perpendicular to $\hat{d}_{1}$ from start point $\left(P_{1}\right)$ to end point $\left(Q_{1}\right) 50 \mathrm{~m}$ on either side of $\hat{S}_{1}$ (Fig 12). These two waypoints are sent to the USV's guidance system, and the vehicle advances at a speed of $1 \mathrm{~ms}^{-1}$ from $P_{1}$ to $Q_{1}$. Every 1 s, the vehicle updates its location and heading estimate, and the coil readings are taken.

Although ideally the vehicle follows the straight line from $P_{1}$ to $Q_{1}$ with its heading aligned along $\overline{P_{1} Q_{1}}$, this is not always the case as there may be surface currents and other environmental effects that distort the vehicle's path. The estimate of the vehicle's

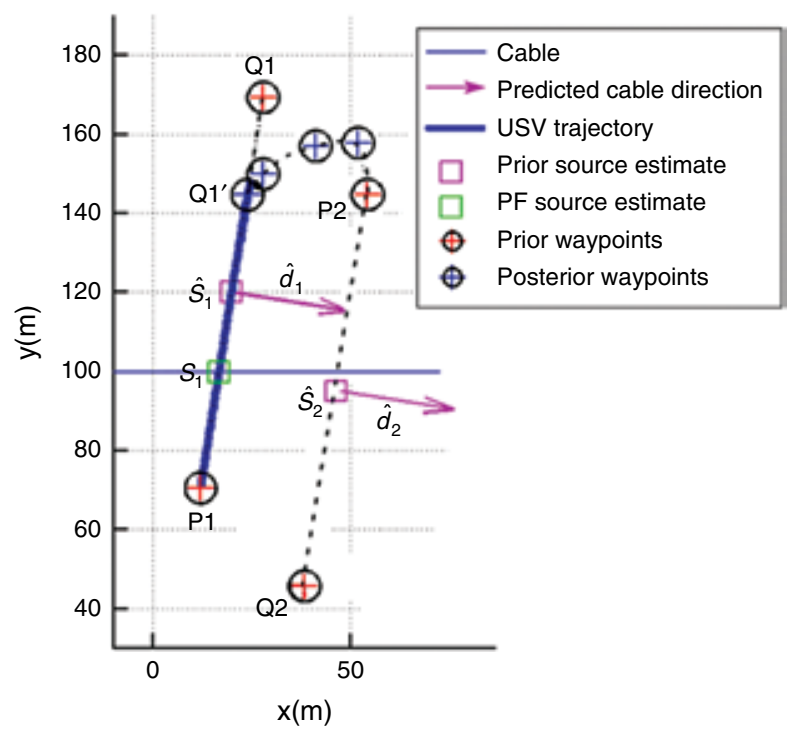

Fig 12: USV trajectory showing cable estimation process

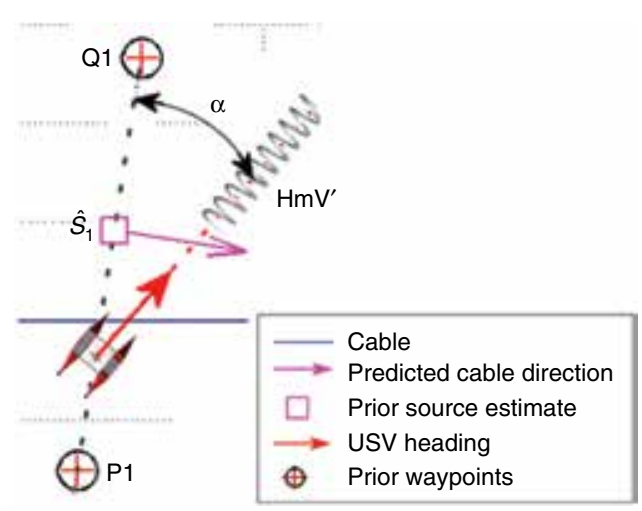

Fig 13: Correction of horizontal coil reading 


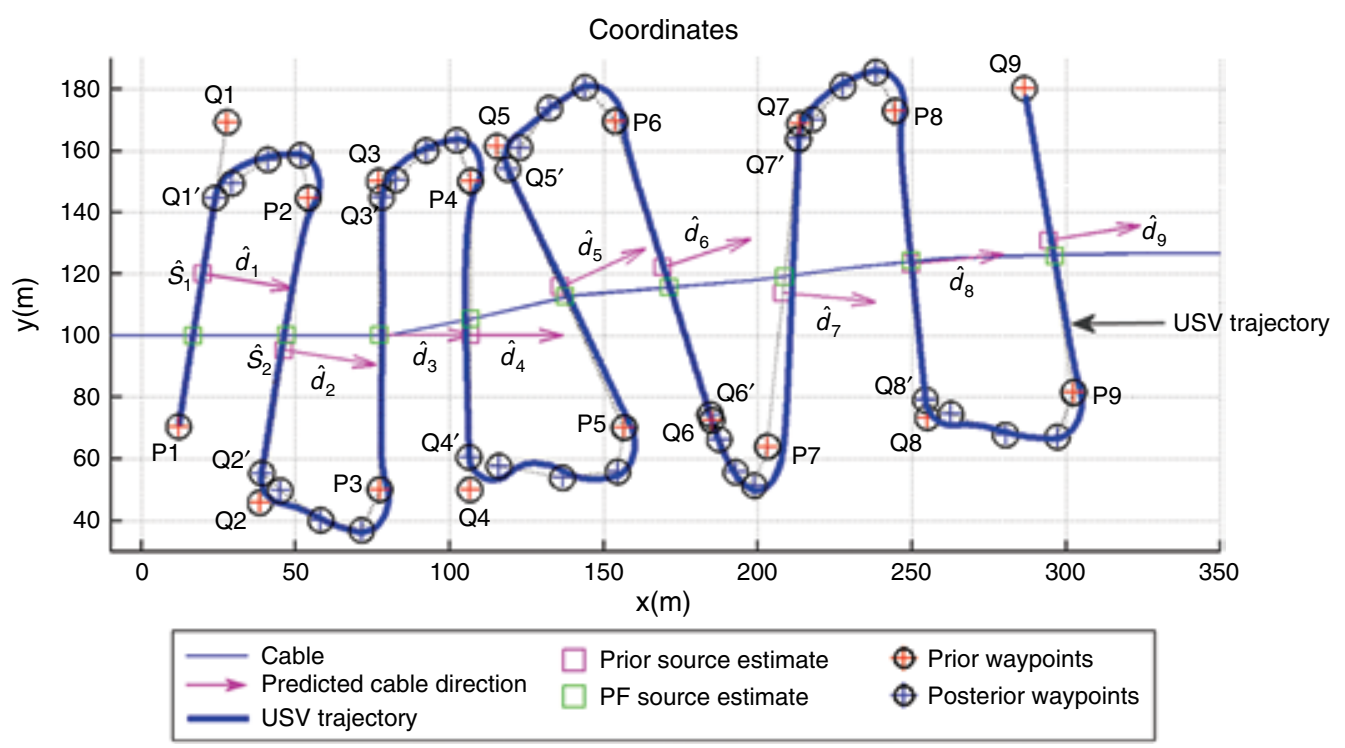

Fig 14: USV trajectory showing complete cable estimation process

heading is thus used to correct the horizontal coil reading of the MF for use by the BPF algorithm, which assumes the horizontal coil to be perpendicular to the cable's direction at the source point $\hat{S}_{1}$. If $\alpha$ is the error between the heading angle of the USV and the direction of $\overline{P_{1} Q_{1}}$ at some instant $k$ (Fig 13), then the measured horizontal coil reading, $\operatorname{HmV}^{\prime}(k)$, is corrected according to Equation 16.

$$
H m V(k)=\frac{H m V^{\prime}(k)}{\cos (\alpha)}
$$

While initially the USV targets the end point $Q_{1}$ of $l_{1}$, when the fuzzy inference system (see section 2) lowers its flag, the coil readings along this line will no longer be meaningful. At this point, the BPF determines an a posteriori or corrected cable source point on $l_{1}$, namely $S_{1}$, based on which an a priori estimate of the position of the source on the next crossover line is obtained as $\hat{S}_{2}=S_{1}+\rho \hat{d}_{1}$, where $\rho$ is a prescribed distance $(30 \mathrm{~m}$ in this case). The estimated direction of the cable at $\hat{S}_{2}, \hat{d}_{2}$, is set equal to $\hat{d}_{1}$. Having estimated $\hat{S}_{2}$ and $\hat{d}_{2} 30 \mathrm{~m}$ downstream, the next crossover line $\left(l_{2}=\overline{P_{2} Q_{2}}\right)$ is projected as a segment perpendicular to $\hat{d}_{2}$ from start point $P_{2}$ to end point $Q_{2}$, spanning $50 \mathrm{~m}$ either side of $\hat{S}_{2}$. There is now no further interest in the vehicle reaching $Q_{1}$, and therefore a new target $Q_{1}{ }^{\prime}$ on $l_{1}$ is established just $10 \mathrm{~m}$ ahead of the vehicle. From this, a path is generated between this new final point on $l_{1}$ and the initial point on $l_{2}\left(P_{2}\right)$ via a Hermite spline, on which several intermediate waypoints are generated to provide a smooth turning path for the USV.

For a general line $l_{i}$, after the $a$ posteriori estimate of the source location, $S_{i}$, has been determined by the BPF algorithm, the a priori estimate of the position of the source on the next crossover line $\left(\hat{S}_{i+1}\right)$ is obtained by extrapolating the last three source estimates $\left(S_{i-2}, S_{i-1}, S_{i}\right)$ with a parabolic function a distance of $\rho$ further downstream. The cable's direction at $\hat{S}_{i+1}, \hat{d}_{i+1}$, is estimated to be that of the tangent to the parabola at that point. A simulation of the cable localisation and tracking process is shown in Fig 14.

Navigational data for said simulation are given in Fig 15. The USV heading estimation was based on the WIKF technique described in the previous section. In order to illustrate the robustness of this technique, an interval model centred around Equations 6 to 8 with $\pm 5 \%$ uncertainty on all of the values was considered. It was assumed that the vehicle's true dynamics was given by the upper boundaries of all the intervals. Estimates of the turning rate and heading angle obtained from the WIKF, an ideal KF (based on the true vehicle dynamics), and a nominal KF (based on the nominal model Equations 6 to 8) are shown in Fig 15a,b. Also shown are the IKF bounds, the true values of turning rate and heading, and the gyro measurements. For completeness, the differential thrust applied by the autopilot is shown in Fig 15c, the innovation sequence of the nominal $\mathrm{KF}$ that is fed to the trained ANN in Fig 15d, and the desired and ANNpredicted weightings for the IKF bounds in Fig 15e.

A quantitative comparison of the turning rate and heading errors of each of these three filters is given in Table 1. Both from the figure and the table, it can be observed how the nominal KF heading estimates are biased because of the incorrect vehicle model assumed. The average heading estimate error of the $\mathrm{KF}$ is almost four times that of the WIKF. 

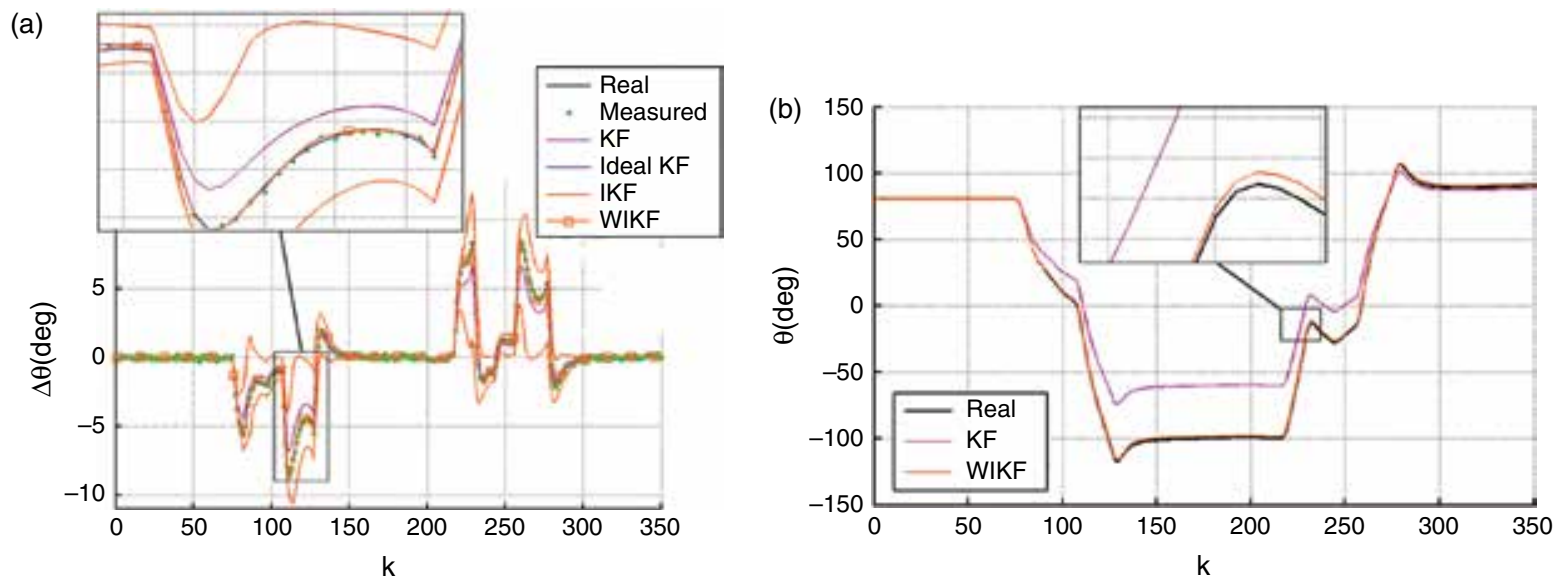

(c)

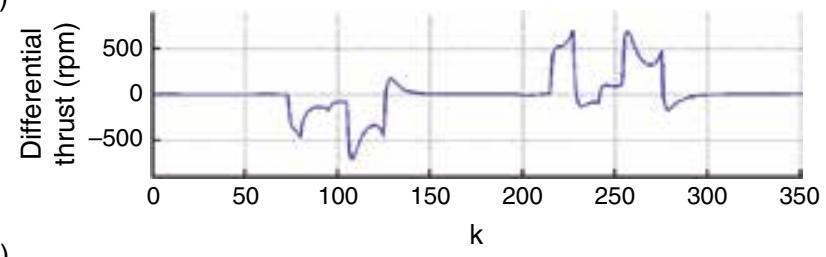

(d)

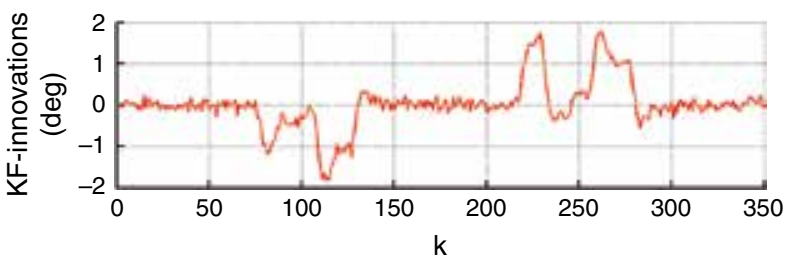

(e)

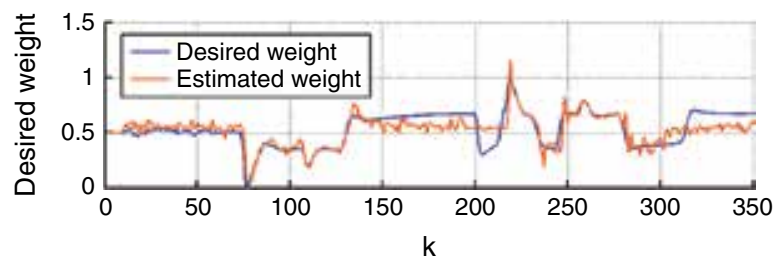

Fig 15: Navigational data for USV autonomous navigation using WIKF heading estimates: (a) USV turning rate; (b) USV heading angle; (c) differential thrust (rpm); (d) KF innovation sequence (deg); and (e) desired weighting sequence for IKF and ANN weight estimation

Table 1: Navigational RMS errors of the three filters

\begin{tabular}{lllc}
\hline & Ideal KF & WIKF & Nominal KF \\
\hline USV yaw rate RMS error (deg/s) & $7.9696 \mathrm{e}-04$ & 0.1478 & 0.5863 \\
USV heading RMS error (deg) & 0.0205 & 6.1234 & 23.3968 \\
\hline
\end{tabular}

Fig 16 shows what the actual trajectory of the USV would be like if these headings were used for navigation, highlighting the importance of accurate heading estimates to minimise deviation from the desired path. Moreover, because of the incorrect heading data, the readings of the MF in the direction of the crossover line would not be obtained correctly, leading to an incorrect estimation of the cable source by the BPF.

\section{Conclusion}

The BPF algorithm for estimating the source of the cable was initially developed for surveys from manned platforms that attempted to estimate the cable's position by successively criss-crossing its assumed path. This technique proved successful in practice as long as the platform was guided adequately. On the other hand, the WIKF was developed to provide the Springer USV with an accurate heading estimation system to enhance autonomous operation of the vehicle.

The present paper has proposed a methodology for autonomously carrying out subsea cable localisation from a USV using the BPF algorithm. To make this possible, an effective dynamic path planning algorithm to guide the USV has been proposed. It was also aided by a fuzzy-logic-based data discrimination 


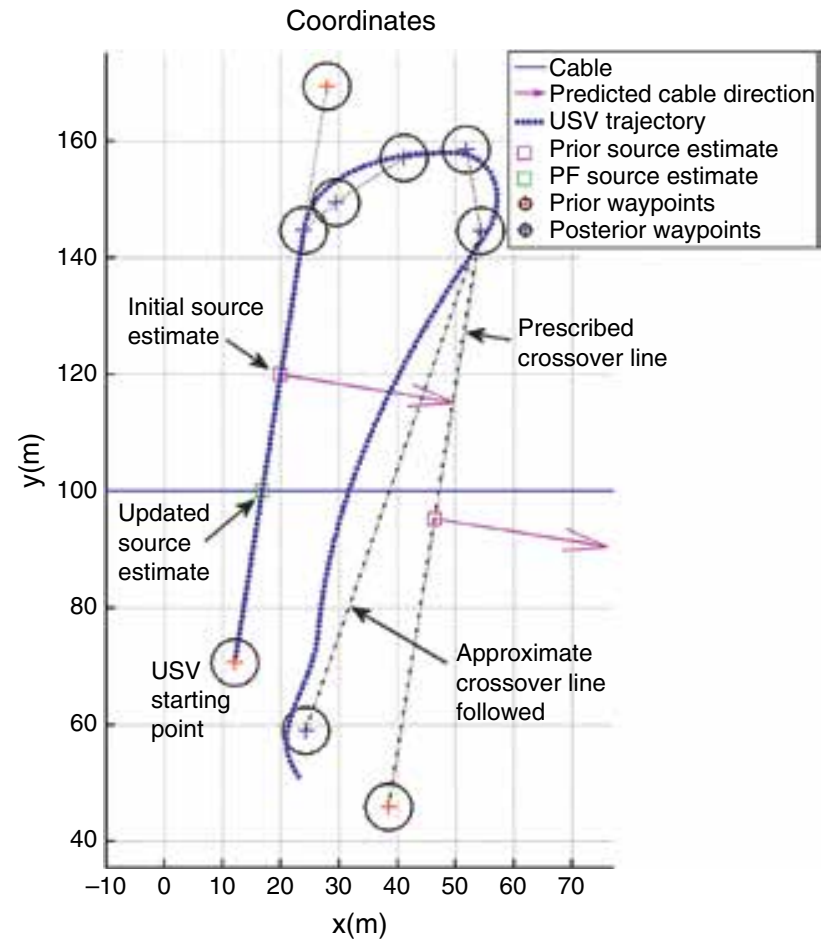

Fig 16: Path followed by the USV based on KF heading estimates

procedure that indicates when meaningful coil readings are being obtained. This allows the USV to move on to the next projected crossover line before completing the initially projected current line, saving time and operation costs.

In addition, the uncertainty and decision-making delays of manned navigation and guidance, which were observed as one of the main practical drawbacks in the cable localisation surveys that were carried out using the BPF, are greatly reduced by the degree of automation proposed in the present paper. From the point of view of accuracy, the robust USV heading estimation based on the WIKF means that the horizontal coil readings are more accurately projected onto the global reference frame. By providing only those coil readings that are actually meaningful to the BPF algorithm, it is able to converge more rapidly and reliably.

The method proposed here does not replicate the cable localisation method carried out from a manned boat, but improves on its effectiveness, reducing time and costs, and of course, without the safety concerns of manned operation. In the simulation shown here, the USV was able to track the cable even though the initial assumption of the source was over $20 \mathrm{~m}$ away from the true source, and the initial assumed cable direction was over 10 degrees off. A limitation to the approach described here is the assumption that the object being tracked does not contain pronounced curvatures, since the projected crossover lines are assumed to be perpendicular to the cable based on a priori estimates of the cable's direction. Nevertheless, this assumption is mostly true for cables and pipelines.

It is envisaged to test this approach in a real cabletracking mission off the Cornish coast in the southwest of England. Furthermore, the method will be extended for the localisation of small ferromagnetic objects, for which MFs will be generated from eddy currents induced by alternating MFs of the searching coils themselves. The resulting induced MF distribution of smaller objects will be shaped in the form of a single peak above the object, for which different path planning routines will need to be developed, as well as the necessary intelligent discrimination methods to identify the type of object localised.

\section{References}

Annamalai A, Motwani A, Sharma SK, Sutton R, Culverhouse P and Yang C. (2014). A robust navigation technique for integration in the guidance and control of an uninhabited surface vehicle. Unpublished data.

Bogie I. (1972). Conduction and magnetic signalling in the sea a background review. Radio and Electronic Engineer 42: 447.

Chen G, Wang J and Shieh LS. (1997). Interval Kalman filtering. IEEE Transactions on Aerospace and Electronic Systems 1: 250-258.

Cowls S and Jordan S. (2002). The enhancement and verification of a pulse induction based buried pipe and cable survey system. OCEANS'02 MTS/IEEE, 508-511.

Crisan D and Obanubi O. (2012). Particle filters with random resampling times. Stochastic Processes and their Applications 122: 1,332-1,368.

Fallon MF and Godsill S. (2010). Acoustic source localization and tracking using track before detect. IEEE Transactions on Audio, Speech, and Language Processing 18: $1,228-1,242$.

Gordon N. (1993). Novel approach to nonlinear/nonGaussian Bayesian state estimation. Radar and Signal 140: 107-113.

Guardian. (2014). UK government gives green light to offshore windfarm. Press Association. Available at www. theguardian.com/environment/2014/jun/17/ukgovernment-gives-green-light-to-offshore-windfarm, last accessed $<31$ January 2015>.

King RW. (1989). Lateral electromagnetic waves from a horizontal antenna for remote sensing in the ocean. IEEE Transactions on Antennas and Propagation 37: $1,250-1,255$.

Kojima J, Kato Y, Asakawa K, Matumoto S, Takagi S and Kato N. (1997). Development of autonomous underwater vehicle AQUA EXPLORER 2 for inspection of underwater cables. In: Oceans '97 MTS/IEEE Conference Proceedings, 6-9 October, Halifax, Nova Scotia.

Motwani A. (2012). A Survey of Unhabited Surface Vehicles. Plymouth: MIDAS Technical Report: MIDAS.SMSE.2012. TR.001. Available at www.tech.plymouth.ac.uk/sme/ springerusv/2011/publications3.html, last accessed $<12$ January 2015 $>$.

Motwani A, Sharma SK, Sutton R and Culverhouse P. (2013). Interval Kalman filtering in navigation system design for 
an uninhabited surface vehicle. Journal of Navigation 66: 639-652.

Motwani A, Sharma SK, Sutton R and Culverhouse P. (2014a). Application of artificial neural networks to weighted interval Kalman filtering. In: Proceedings of the Institution of Mechanical Engineers, Part I: Journal of Systems and Control Engineering, vol. 228, 267-277

Motwani A, Sharma SK, Sutton R and Culverhouse P. (2014b). Computation of stable interval Kalman filter bounds for their use in robust state estimation for an uninhabited surface vehicle with bounded indeterminate system dynamics. In: IEEE Intelligent Vehicles Symposium Proceedings. 8-11 June, Dearborn, Michigan, USA, 356-361.

Naeem W, Xu T, Chudley J and Sutton R. (2006). Design of an unmanned surface vehicle for environmental monitoring. In: Proceedings of World Maritime Technology Conference, 1 March, London, UK, 1-6.

Simon D. (2006). Optimal State Estimation: Kalman, H-infinity, and Nonlinear Approaches. Hoboken, New Jersey: John Wiley \& Sons, 552pp.
Szyrowski T, Sharma SK, Sutton R and Kennedy GA. (2013a). Developments in subsea power and telecommunication cables detection: Part 1 - Visual and hydroacoustic tracking. Underwater Technology 31: 123-132.

Szyrowski T, Sharma SK, Sutton R and Kennedy GA. (2013b). Developments in subsea power and telecommunication cables detection: Part 2 - Electromagnetic detection. Underwater Technology 31: 133-143.

Szyrowski T, Sharma S, Sutton R and Kennedy GA. (2014). Point localisation of a subsea cable using particle filters. In: Proceedings of UKACC International Conference on Control (CONTROL), 9-11 July, Loughborough, UK.

Takagi S, Kojima J and Asakawa K. (1996). DC cable sensors for locating underwater telecommunication cables. OCEANS ' 96 MTS/IEEE Conference Proceedings. The Coastal Ocean - Prospects for the 21st Century, 339-344.

Tumanski S. (2007). Induction coil sensors - A review. Measurement Science and Technology 18: R31-R46.

Won I. (2003). Characterization of UXO-like targets using broadband electromagnetic induction sensors. IEEE Transactions on Geoscience and Remote Sensing 41: 652-663. 